\title{
COPLINK Agent: An Architecture for Information Monitoring and Sharing in Law Enforcement
}

\author{
Daniel Zeng, Hsinchun Chen, Damien Daspit, Fu Shan, Suresh Nandiraju, \\ Michael Chau, Chienting Lin \\ Department of Management Information Systems \\ University of Arizona, Tucson, Arizona 85721 \\ zzeng, hchen, damien, shan, mchau, linc $\} @$ eller.arizona.edu
}

\begin{abstract}
In this paper, we report our work on developing and evaluating a prototype system aimed at addressing the information monitoring and sharing challenges in the law enforcement domain. Our system, called COPLINK Agent, is designed to provide automatic information filtering and monitoring functionalities. This system also supports knowledge sharing by proactively identifying officers who are working on the same or similar cases on a real-time basis. To accommodate the mobile needs of law enforcement officers who are constantly in the field, COPLINK Agent can deliver messages through a variety of communications channels including e-mail, pager, and mobile phones. In order to assess the effectiveness of COPLINK Agent, we conducted a pilot user study at the Tucson Police Department. Overall, COPLINK Agent was shown to be an effective tool for improving the effectiveness and efficiency of criminal investigations in crimes such as gang, theft, and fraud.
\end{abstract}

\section{Introduction}

The rapid advancement of information technologies and the Internet provides great opportunities as well as challenges for government agencies. These technologies not only allow easier and faster access to information but also facilitate the sharing and reuse of information. In the law enforcement domain, information access is especially critical for crime analysis and investigation. Consequently, police officers and investigation personnel are increasingly becoming knowledge workers whose daily activities include considerably complex and extensive interactions with diverse information and knowledge sources. The types of interactions include: selecting, collecting, preserving, organizing, using, accessing, analyzing, and producing data. In addition, timely access to information is often critical. Consequently, crime and police report data are rapidly migrating from paper-based records to automated law enforcement records management systems (RMS). Despite the availability of such viable RMS solutions, there are still several major issues and challenges not yet addressed by existing systems. First, crime analysts often need to query different distributed data sources, including both internal databases as well as external ones managed by other law enforcement agencies. These data sources, funded and maintained by multiple agencies, often employ a wide range of different hardware platforms, database sys- 
tems, network protocols, data schemas, and user interfaces. To find the desired information, law enforcement personnel have to know where such data sources are located and how to access them. As such, a large amount of manual and cognitive effort is required to query all the relevant data sources, each with a different search interface.

Another major problem concerns the dynamic nature of law enforcement data sources. Since many cases involve long periods of investigation, law enforcement personnel often have to track the activities of a particular suspect or the whereabouts of a vehicle over a long period of time. As the data are updated frequently, the database has to be repeatedly queried for changes, often on a daily basis. Since such automatic monitoring functions are not available in most current systems, the data sources have to be checked manually, requiring a lot of time and effort from the user. Conceivably, it is not uncommon that cases requiring constantly monitoring have to be dropped because of lack of resources. Last but not least, the lack of support for knowledge sharing is yet another problem confronting the current law enforcement information systems. From their daily work, law enforcement personnel with different job functions and working at different locations can easily acquire a vast amount of knowledge about a particular suspect or case. Such knowledge, nonetheless, is tacit and often not efficiently shared. When a police officer needs some particular information, it is often not clear whom to contact. It is common that two different law enforcement units are working on two closely related cases (e.g., related to the same person) without knowing each other's existence or progress. Naturally, the two units are not able to collaborate and share their collective knowledge. Thus, the ability to share knowledge in a collaborative environment by linking together people who are working on the same or similar cases can significantly improve law enforcement agencies' crime-fighting capabilities.

In this paper, we report on our experience in designing and evaluating a collaborative information monitoring system for law enforcement. This approach was implemented in a prototype system called the COPLINK Agent. COPLINK Agent was designed to answer the aforementioned challenges by providing automatic information filtering and knowledge sharing functionalities. The rest of the article is outlined as follows. Section 2 reviews related research and existing systems in the law enforcement domain. Section 3 discusses the research questions outlined in this study. Section 4 describes the system architecture and main components of the COPLINK Agent. In Section 5, a sample user session with the system is described in detail to illustrate its use in a law enforcement environment. Section 6 focuses on a user study designed to evaluate the COPLINK Agent and answers the research questions raised above. We conclude the paper in Section 7 by summarizing our research contributions and suggesting future research directions.

\section{Research Background}

\subsection{Information Systems in the Law Enforcement Domain}

Quick and easy access to information is critical to the success of law enforcement agencies. Database technologies have been widely used to manage crime and police 
reports to provide faster and easier access for law enforcement personnel $[15,18]$. One such example is the COPLINK Connect system [7,14]. COPLINK Connect aims to enable law enforcement agencies to search for information more effectively by providing an user-friendly interface that integrates data from various sources such as incident records, mug shots, and gang information. COPLINK Connect was deployed in 2001 at the Tucson Police Department (TPD), which has more than 1,000 employees and serves a population of over 475,000 citizens. This system has been shown in a field study to have improved the efficiency of detectives and crime analysts [14].

Other information technologies also have been used in law enforcement. For example, the Comstat system introduced by the New York Police Department uses computer statistics and crime mapping techniques to identify the types of crimes happening in different districts [10]. Another example, COPLINK Detect system, uses co-occurrence analysis to identify the relationships among different entities (e.g., persons, vehicles, locations, and organizations) in criminal justice databases [14]. Data mining techniques have also been applied to identifying interesting patterns in criminal data. For example, a self-organizing map is used to cluster similar sexual offense cases into groups in order to identify serial offenders [1].

\subsection{Information Monitoring and Sharing}

The law enforcement information systems discussed above have mainly focused on two aspects, i.e., providing easy and efficient access to data, as well as performing analysis on existing data. However, none of these systems support automatic information monitoring or information sharing among users, which have been widely studied for Web applications. There are many monitoring and notification systems for Web information sources. One example is the NorthernLight Web search engine (www.northernlight.com), which alerts users when new Web pages are added to the database. Some client-side search tools, such as Copernic Agent (www.copernic.com) and WebSeeker (www.bluesquirrel.com), also provide the functionality for scheduling automatic searches. Stock prices are also frequently monitored. For example, E*trade (www.etrade.com) allows users to choose which stocks they want to monitor and the users are alerted when the stock price reaches the level they specified. In the financial application arena, more advanced monitoring (e.g., monitoring based on the results of complex financial analysis) has also been proposed [24]. In these systems, users can often opt to be alerted in different ways, such as Web messages, emails, pagers, voice messages, or short messages for mobile devices. In the area of providing monitoring and alerting support for law enforcement applications, the FALCON system [4] developed at Charlotte-Mecklenburg Police Department (CMPD) in Charlotte, North Carolina offers the functionality of monitoring all incoming police records as well as sending alert messages to police offers by email and pager. FALCON, nonetheless, does not offer collaborative filtering capabilities or advanced collaboration functions.

To facilitate user collaboration and information sharing, collaborative filtering, also referred to as recommender system, has been widely studied in Web applications. Goldberg et al. [12] defines collaborative filtering as a kind of collaboration in which people help one another perform filtering by recording their reactions to documents 
they read. Examples of collaborative filtering and recommender systems include Amazon.com, GroupLens [17], Fab [3], Ringo [22], Do-I-Care [23], and Collaborative Spider [6]. When a user performs a search, these systems will recommend a set of documents or items that may be of interest based on this user's profile and other users' interests and past actions. Collaborative filtering systems typically operate in a "push" mode. When certain users come across some interesting pieces of information, they highly recommend these pieces, or "push" them to other users who share similar interests. For instance, Amazon.com uses collaborative filtering to recommend books to potential customers based on the preferences and recommendations of other customers who have similar interests or purchasing histories. Annotations in free-text or predefined formats are also incorporated in systems such as AntWorld [16], Annotate! [11], and CIRE [21] to facilitate collaboration among users. In collaborative filtering systems, one major issue is users' willingness to share information. It has been suggested that Lotus Notes was not well utilized because users had little or no incentive to share information [20]. The situation, however, becomes less problematic for Web searching, which consists mostly of voluntary contributions [21] as users are more willing to contribute in exchange of pride and popularity. Lastly, many systems attempt to minimize user effort by capturing user profiles/patterns automatically [2,23].

\section{Research Questions}

As discussed earlier, information monitoring and information sharing are two areas that are critical to criminal investigation and analysis. Although these topics have been widely studied in other areas such as Web applications/computing, they have not been adequately applied to address the unique problems in law enforcement. In our research, we aim to answer the following research questions:

- Can information monitoring and sharing techniques be effectively applied to existing law enforcement information systems?

- Can such a system alleviate the existing information monitoring and sharing problems in the law enforcement domain?

- How will such a system affect law enforcement personnel in their crime analysis and investigation work? Can the system improve effectiveness and efficiency of current criminal investigation practices?

\section{COPLINK Agent System Architecture}

\subsection{System Architecture Overview}

Attempting to answer the above questions, we propose a modular, personal-agentoriented architecture to support information monitoring and collaboration in law enforcement. Based on the architecture, we implemented a prototype system called COPLINK Agent, which is built on top of the COPLINK Connect system discussed in Section 2.1. The COPLINK Connect system, which supports data access and basic 
searching/sorting functions, serves as an ideal test environment for the proposed architecture as we can readily add advanced monitoring, collaboration, and alerting functions to the system.

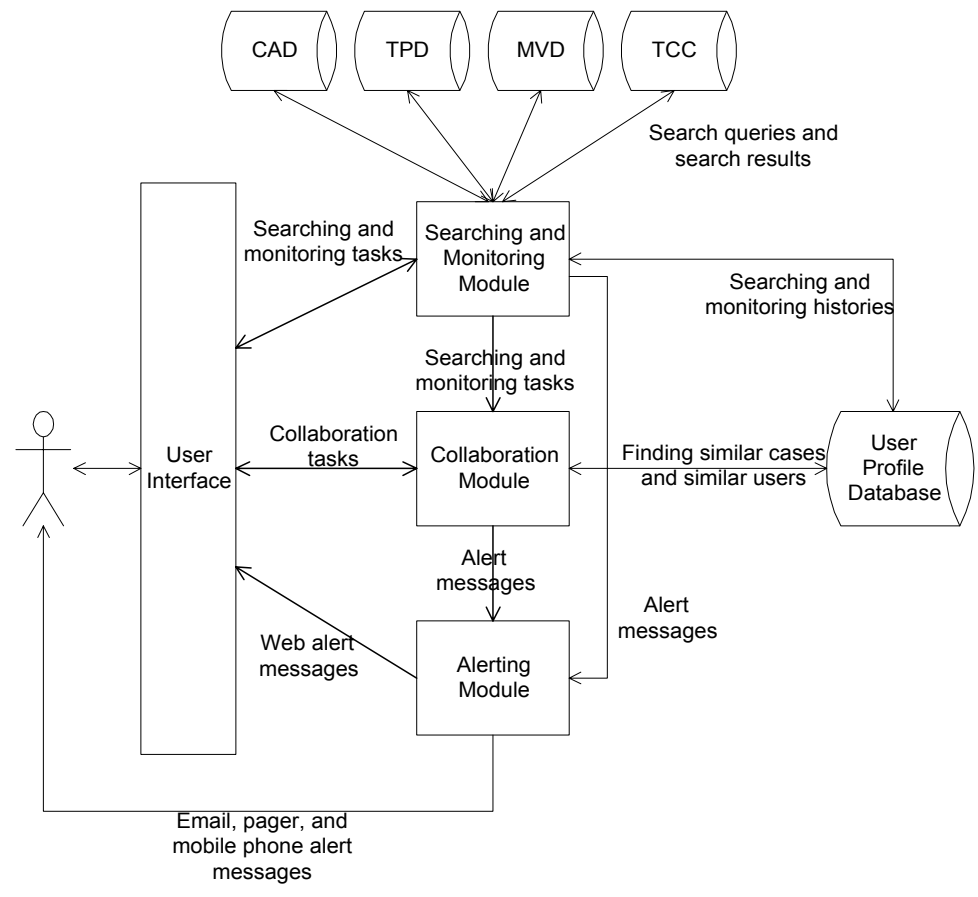

Fig. 1. System architecture for COPLINK Agent

Our proposed architecture is shown in Figure 1. It consists of a Web-based user interface and three functional modules, namely the Searching and Monitoring Module, the Collaboration Module, and the Alerting Module. The Searching and Monitoring Module is responsible for retrieving records from the database, keeping a list of monitoring tasks for each user, and performing these tasks periodically based on the user's preference. The Collaboration Module facilitates the sharing of information among different users. The Alerting Module is responsible for keeping track of the messages for each user and delivering these messages through different communications channels. The Personalization Module keeps track of each user's search history and allows the user to customize various system settings. The functionalities of each module are described in detail below.

\subsection{Searching and Monitoring Module}

The Searching and Monitoring Module accepts search queries from users and forwards them to the corresponding data sources. In addition to the COPLINK database 
for TPD data used in COPLINK Connect, the Searching and Monitoring Module connects to three additional data sources: the Computer Aided Dispatch (CAD) database used at TPD, the Motor Vehicle Division (MVD) database in the state of Arizona, and the Tucson City Court (TCC) Web-based search engine. These databases provide additional person, location and vehicle information that are not available in the COPLINK database.

In addition to the search functionalities, this module also allows users to set up monitoring tasks for the available data sources. For instance, if a user wants to monitor all four data sources for a particular query, the monitor task will be stored in the user profile database and the data sources will be automatically monitored for changes. Different mechanisms are used to monitor the data sources due to the differences in their nature. The COPLINK database, to which our system has full access, is monitored by adding triggers to the database directly. For external databases, such as the CAD and the MVD databases, the Searching and Monitoring Module sends periodic queries to these databases. For TCC, which is accessed by a Web-based search form, the system sends HTTP request to periodically query the search engine. When the relevant records are updated or inserted into the databases, the system will send an alert message to the user through the Alerting Module. Readers are referred to Zeng et al. [25] for detailed technical discussion on how monitoring requests are handled by COPLINK Agent. To facilitate the management of user requests, the searching and monitoring sessions are also stored in the user profile database. Every time a user logs on the system, he/she can retrieve the previous searches and monitor histories from the user profile database. The user can then review previous search sessions as well as edit the settings of existing monitoring tasks.

\subsection{Collaboration Module}

To facilitate collaboration among law enforcement personnel, we developed a collaborative filtering module in COPLINK Agent. While traditional collaborative filtering relies on documents read (e.g., [17]) or items purchased by users (e.g., Amazon.com), we make use of the users' search actions and search histories. The rationale behind this design is that when two users search for the same information in criminal databases, it is likely that the users have similar information needs and that they may possibly be working on two related cases. By storing and analyzing user search histories, the Collaboration Module facilitates such collaboration in two different ways. First, when a user performs a search, the Collaboration Module can instantly identify other users who have performed a similar search in the past. For example, if a detective runs a search on a particular suspect, he/she can also view all the other users who have searched information about this suspect. Second, the user also can specify whether he/she wants to be notified when some other users perform a similar search in the future. When this happens, the Collaboration Module will notify both users through the Alerting Module. The users can then contact each other and determine whether they have any information to share. Currently, we consider two searches to be similar only if the search query terms match exactly with each other. Other matching algorithms can be easily added and will be pursued in the future. 
In our initial user requirement study with TPD, detectives and crime analysts stated that it is very important to protect the confidentiality of the police personnel and the cases on which they are working [5]. While it is safe and possibly beneficial to share a user's search history in some cases, in other cases users have to keep their search histories confidential and not accessible by other users (e.g., cases that involve undercover or internal investigation). It is also important that the system does not send an overwhelming number of alert messages to the users, which would otherwise create yet another information overload problem. To cater to the different levels of confidentiality requirement and information needs, the Collaboration Module allows user to specify the confidentiality level and the alerting level of each search. More details about the different levels will be discussed in Section 5.2.

\subsection{Alerting Module}

The Alerting Module manages all the alert messages that should be sent to a user. Whenever a user sets up a task in the Searching and Monitoring Module or the Collaboration Module that may result in future alerting messages, the user can specify how he/she wants to be notified. When an alerting condition is satisfied, the Alerting Module will receive the alerting messages from the collaboration and search modules. The messages will then be saved in the database and delivered to the user via the medium specified. Currently, messages can be sent to a user instantly through e-mail, pager, and mobile phone. If the user is currently logged on the system, the message also can be presented through the User Interface. Otherwise, the user can see the message next time he/she logs on the system.

\section{Sample User Sessions with COPLINK Agent}

In this section, we present a scenario in which users utilize the different functions of the COPLINK Agent system.

\subsection{Searching and Collaborating}

Suppose the user wants to perform a search for a person, he can click on the tab "Perform New Search". Currently, four types of searches have been implemented in the system, namely "Person/Organization Search," "Vehicle Search," "Location Search," and "Incident Search." All the search forms have a similar layout while each form has its specific search fields. Person search is used in our example. After the user clicks on "Person Search," the corresponding search form will be shown (see Figure 2).

The search form shown in Figure 2 is divided into the five input areas. The following shows how the user goes through each area and inputs the necessary information.

1) Database Selection: This allows the user to select which data sources are to be searched. In the example shown in Figure 2, the TPD database is chosen by the user. 


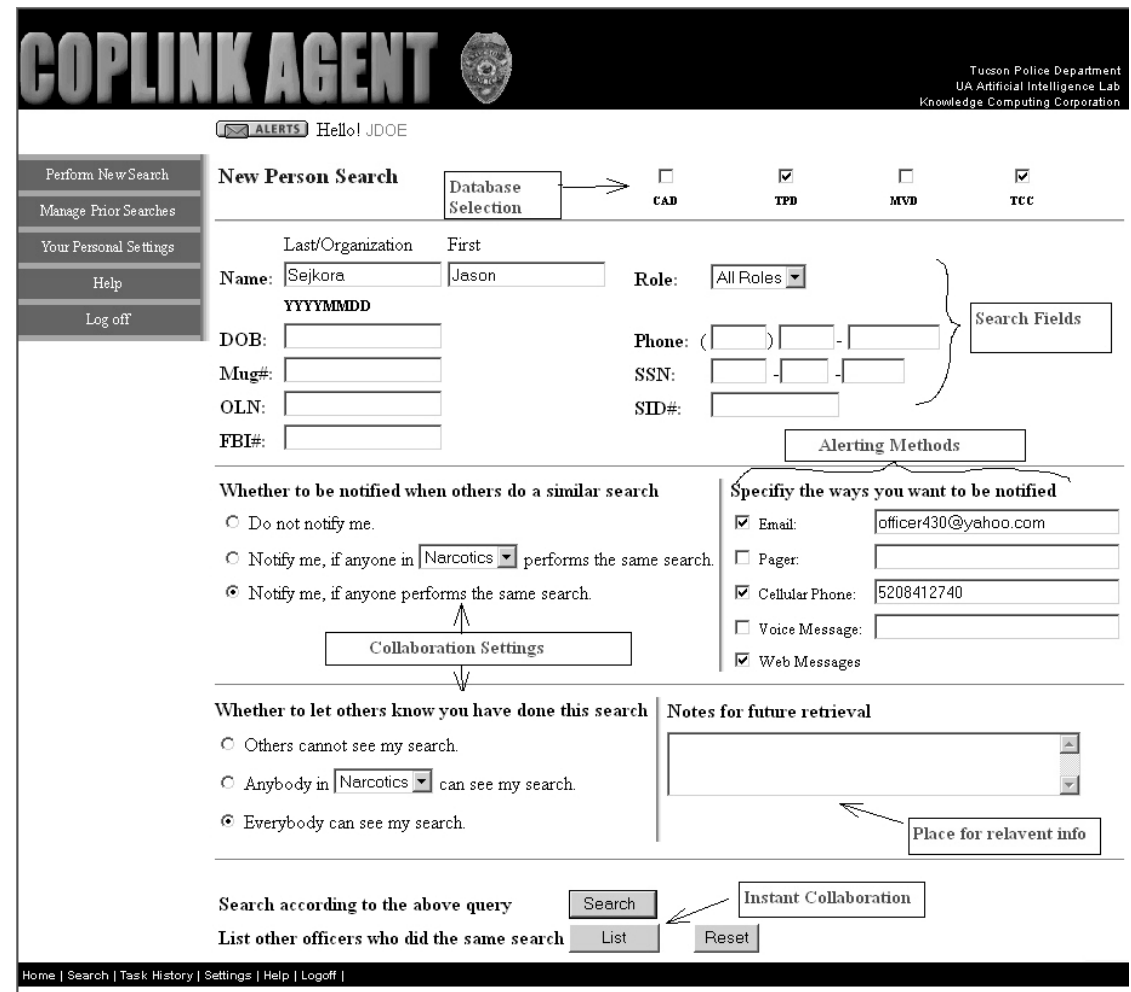

This is a Prototype System. All data are fietitious.
Copyright 82000 UA Antrifial Intelligence Lab and Knowledge Computing Corporation. All Rights Reserved.
All trademaths mentioned herein belong to their respective ounners.

Fig. 2. Person search and monitoring screen for COPLINK Agent

2) Search Fields: The user can then enter the searching criteria. The user wants to search for the records of a person named "Jason Sejkora", so the user enters "Jason" in the first name field and "Sejkora" in the last name field.

3) Collaboration Settings: The user can set the desired level of collaboration. In the upper portion, the user can choose the "Notification Level" of the search. The user can choose to be notified when anyone performs the same search, when anyone in the specified unit performs the same search, or not to be notified at all. In the lower part of the same interface, the user can choose the "Confidentiality Level" of the search. The user can choose to make the search visible to all other users, only users in the same unit, or nobody at all. In this example, the user chooses to be notified if any other user performs the same search. He also chooses to make his search visible to all other users.

4) Alerting Methods: This allows the user to specify how he/she wants to be notified if there are some other users who have performed the same search. Multiple methods can be specified. The user can also decide whether to receive notification through email, cellular phone messages, or Web messages. 
5) Notes: The user can enter some personal notes in this area that are relevant to the search. The notes will be displayed in the alert messages.

After specifying all the information, the user can now perform a search by clicking the "Search" button at the bottom of the search form. The search query is then forwarded to the specified database(s) and the search results are displayed to the user. When the number of search results is large, the user can click on the heading of any column to sort the records based on the values of that column. Alternatively, before performing the search, the user can also click on the "List" button to see whether any other user has performed the same search before. This is called the "Instant Collaboration" function, which allows the user to consult other officers instantly to see whether there is any further information about the person being searched. When the user clicks on the "List" button, a screen will be displayed showing all the users who have performed the same search in the past. The user can then click on the name of any of these users to retrieve their contact information and contact them directly.

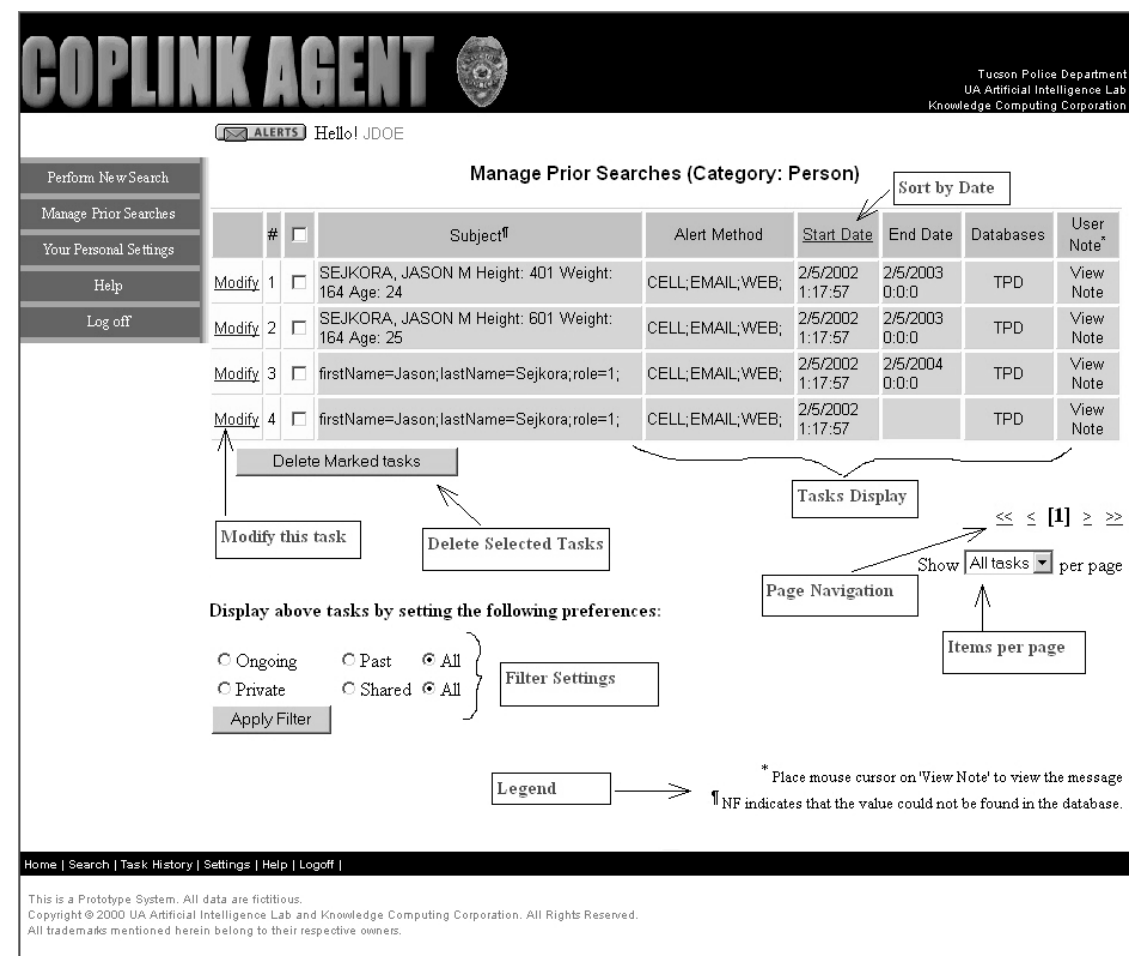

Fig. 3. Managing search and monitoring tasks

\subsection{Information Monitoring}

At the search result screen, the user can choose to add a monitor to the search results. There are two types of monitoring: the user can choose to monitor the changes to the 
existing records shown in the search results, or to monitor any future addition to the database that match the original search criteria. In this example, the user chooses to perform both types of monitoring. As with the collaboration function, the user can also specify how he/she wants to be notified when there is change in the database that matches with the monitoring task. After the alerting method is set, the user can then click on the "Add Monitor Tasks" button and a confirmation message will be displayed.

\subsection{Managing Search Sessions}

When the user logs on the system at a later date, he/she can retrieve all his previous search sessions by choosing the "Manage Prior Searches" tab on the left-hand side menu. All the previous search sessions by the user will be displayed (see Figure 3). The user can choose to review these searches, or modify the monitoring settings of each of these sessions. For cases that have been solved, the user can also delete these search sessions and monitoring tasks from his profile such that he will not be alerted by future changes in the database.

\section{Evaluation}

In conjunction with the Tucson Police Department (TPD), we evaluated the effectiveness of COPLINK Agent by conducting a usability study in the summer of 2002. Details of our evaluation design and analysis follow.

\subsection{Methodology}

Our methodology for evaluating the COPLINK Agent system is a case study method incorporating structured interviews, usability surveys, and archival records analysis (e.g., summary of user-added monitoring tasks and the alerts produced by the system). To select the required usability evaluation techniques, we first identified two usability goals and the three dimensions of usability. The resulting usability metrics [9] encompassing the specific measures and techniques used is shown in Table 1:

Table 1. Usability Metrics of COPLINK Agent Usability Evaluation

\begin{tabular}{|l|l|l|l|}
\hline $\begin{array}{l}\text { Usability } \\
\text { Objective }\end{array}$ & $\begin{array}{l}\text { Effectiveness } \\
\text { Measures }\end{array}$ & $\begin{array}{l}\text { Efficiency } \\
\text { Measures }\end{array}$ & $\begin{array}{l}\text { Satisfaction } \\
\text { Measures }\end{array}$ \\
\hline $\begin{array}{l}\text { Suitability for } \\
\text { Investigative } \\
\text { Tasks }\end{array}$ & $\begin{array}{l}\text { Percentage of Alerts } \\
\text { deemed Useful (Ar- } \\
\text { chival Data + Inter- } \\
\text { view) }\end{array}$ & $\begin{array}{l}\text { Time required to } \\
\text { create a new moni- } \\
\text { toring profile (Inter- } \\
\text { view) }\end{array}$ & $\begin{array}{l}\text { Rating scales for } \\
\text { overall usability } \\
\text { (Survey) }\end{array}$ \\
\hline Learnability & $\begin{array}{l}\text { Percentage of func- } \\
\text { tions learned (Sur- } \\
\text { vey) }\end{array}$ & $\begin{array}{l}\text { Time to learn crite- } \\
\text { ria (Interview) }\end{array}$ & $\begin{array}{l}\text { Rating scales for } \\
\text { ease of learning } \\
\text { (Survey) }\end{array}$ \\
\hline
\end{tabular}


The structured interviews for the pilot users were guided by the COPLINK Agent system $\log$ files which include lists of monitoring profiles that the pilot users added into the system, as well as the alerts that the users received after matches are found. The subjective measure of user satisfaction was evaluated using a standard usability survey instrument. User comments for database monitoring and collaboration functions were also collected, along with suggestions for interface and functionality improvements. Lastly, the qualitative data obtained from the interview sessions was triangulated with the quantitative results from the alert log ratings and usability surveys.

\subsection{Participants}

Fifteen detectives from TPD's Criminal Investigation Division (CID) were recruited to evaluate the COPLINK Agent prototype. The target participants are users who have had extensive prior experiences in the COPLINK Connect systems. The participant profile is shown in Table 2:

Table 2. Participant demographics

\begin{tabular}{|l|l|}
\hline Job Classification & Sergeants $7 \%$, Detectives $80 \%$, Crime Analysts $13 \%$ \\
\hline Police Units & $\begin{array}{l}\text { Gang } 34 \% \text {, Fraud } 20 \%, \text { Theft } 13 \% \\
\text { Robbery } 13 \% \text {, Sex Offense } 20 \%\end{array}$ \\
\hline Gender & Female: $27 \%$, Male $73 \%$ \\
\hline
\end{tabular}

\subsection{Data Collection Procedures}

Participants who received alerts were given listings of the alerts and were asked to rate the usefulness of each alert. Based on the alert ratings and the list of monitoring tasks, a user was asked to provide his or her subjective rating of the alerts received, along with other relevant contextual information including: the nature/type of the case, the search parameters in which a user is interested (Last name, First name, Day of Birth, Race, and Sex, ...etc), the reasons behind adding a monitoring profile, the usefulness of the alert messages received by the users if there is any, and if there is any follow-up done by the user for a particular alert.

Participants were also asked to rate the effectiveness and efficiency of database monitoring and collaboration functions, as well as desired new functionalities. Suggestions for improving current functions and interface were also collected. To gauge subjective user satisfaction, we modified the QUIS instrument as reported by Chin [8] based on our application characteristics and added sections to gauge the effectiveness and efficiency of the monitoring and collaboration functions. QUIS was chosen because it provides specific ratings on the following two specific types of system quality information that we were interested in: (1) overall reactions; and (2) four specific interface factors including screen layout and sequence, terminology and system information, learning factors, and system capabilities. 


\subsection{Summary of Evaluation Study}

Effectiveness and efficiency of COPLINK Agent. During our three-month testing period, a user, on average, received 5.5 alerts per month. Out of those alerts received, approximately $32 \%$ of them were rated equal or above "Somewhat Useful" on our scale. The user's subjective ratings of the alerts also averaged 5.5 out of a 7-point scale (with 7 being the most useful), suggesting a relatively high user satisfaction. The most typical reasons that users add monitoring tasks include: 1) person monitoring: monitoring a suspect, a witness, or an informant, or someone who is on parole; 2) address monitoring: monitoring the exact address or the address of the apartment complex of a suspect; 3) license plate monitoring: monitor a specific car whose license plate number is of interest to the detectives. As to the monitoring and collaboration functionalities, users were generally pleased with the system's capabilities for assisting criminal investigations. One user commented, "Although I only have it 'watching' for 2 names, the information I have received back was instrumental in making at least 2 felony cases that will be prosecuted on the federal level." Another user also commented, "Investigating gangs requires extensive use of networking and sharing of information. I find this option valuable."

In terms of overall effectiveness, COPLINK Agent also garnered positive feedback and one recent success story: A crime analyst involved in our evaluation study had previously added a CAD monitoring task for a particular fraud suspect. One day she received an alert about her suspect using counterfeit money in a local convenient store, and was able to follow up with the case and obtained the video tape from the store's surveillance camera. The alert has led to two felony charges for the criminal on a federal level. Had the crime analyst not received the CAD alert in a timely manner, she would have to wait for at least another 3-4 of weeks to see the case report. By that time, the critical video tape would have been destroyed as the convenient store only keeps video tapes for the past 30 days. As to the efficiency of operating the COPLINK Agent system, most users were able to finish adding a new monitoring profile within 2-5 minutes.

User Satisfaction of COPLINK Agent. The short-form of the QUIS instrument averaged 5.5 for 27 items on a 7-point Likert scale (7: most useful). After conducting a profile analysis, the weaknesses of COPLINK Agent include: lack of help messages, difficult for inexperienced users, and obscure user preference settings. The strengths of COPLINK Agent include: offers good investigative power, easy to read layout, potential for collaborative information sharing, CAD Integration, as well as high intention to use. We were able to use the feedback on the user satisfaction to create a list of system enhancements that we plan to implement in the next phase of the COPLINK Agent development.

\subsection{Discussions}

In this section, we report the lessons learned based on our field study of COPLINK Agent in TPD's criminal investigative units. First, when asked to compare alerts originating from the TPD database and CAD, most detectives indicated that CAD alerts are more valuable because of their timeliness. A significant portion of the alerts 
from the TPD database were in fact CAD records that were eventually entered into the RMS system, albeit with a 3-4 weeks delay. Although TPD's CAD records are examined once per day by the COPLINK Agent system, 50\% of the users expressed interest in allowing CAD entries to be monitored on a nearly real-time basis (e.g., at least every 10 minutes or every hour). Some user comments in this area include: "The only other improvement I could ask for would be it query a couple times a day as opposed to once every 24 hours." Another user states, "Detective could have been dispatched immediately, if notification had been in real time."

Second, when most detectives receive an alert originating from CAD, they typically immediately contact the field officers directly to inquire about the nature and circumstances of the case. The system essentially becomes an effective tool for linking people together according to O'Leary's knowledge management framework [19]. Specifically, CAD alerts in COPLINK Agent facilitate interunit knowledge sharing [13] by creating a short and direct network path from the detective units to the field officers who possess the requisite knowledge pertinent to the cases at hand. One user commented about the usefulness of CAD alerts by saying, "COPLINK Agent is allowing us to respond to incidents we know are important that the field units perhaps don't realize in a timely manner." Another user also echoed the same assessment: "COPLINK Agent is good because so many times we complain that we don't get information from the field. This way I know who ran (a query in our database on) someone and can inquire as to why."

\section{Conclusions and Future Directions}

In this paper, we report our work on designing and evaluating a prototype system for information monitoring and sharing in the law enforcement domain. The system, called COPLINK Agent, is based on an Agent system architecture designed to provide automatic information filtering functionalities and facilitate knowledge sharing between police personnel. Through the interactions between the searching, collaboration and alerting modules, COPLINK Agent can help alleviate the information overload problem faced by many police officers and detectives. COPLINK Agent has also been shown to be an effective tool for improving the effectiveness and efficiency of criminal investigations in areas such as gang, theft, and fraud cases. Given the encouraging results from the user study, we plan to incorporate the functionalities of the COPLINK Agent system into the latest COPLINK Connect system deployed at TPD. Afterwards, a larger scale testing will be performed to study how the number of users affects the usability of the system. Additionally, we continue to work on improving each individual component of the COPLINK Agent system. Specifically, we are working on supporting more alerting methods, as well incorporating several loadbalancing and task scheduling algorithms in the monitoring module such that the monitoring tasks can be scheduled effectively to avoid overloading particular databases. Load balancing techniques will gradually become more important as the number of users increases. We are also enhancing the collaboration module by adding fuzzy match techniques that can be used to identify similar searches. Lastly, we plan 
to apply data mining techniques on the user profile database in order to group similar users together and analyze their search patterns.

\section{Acknowledgement}

The work described in this report was substantially supported by the following grants: (1) NSF Digital Government Program, "COPLINK Center: Information and Knowledge Management for Law Enforcement," \#9983304, July 2000-June 2003; (2) National Institute of Justice, "COPLINK: Database Integration and Access for a Law Enforcement Intranet," July 1997-January 2000; (3) NSF/CISE/CSS, "An Intelligent CSCW Workbench: Personalization, Visualization, and Agents," \#9800696, June 1998-June 2001. We also would like to thank all of the personnel from TPD who participated in this study. In particular, we would like to thank Lt. Jenny Schroeder, Det. Tim Petersen, and Dan Casey. Lastly, we also would like to thank members of the COPLINK Team at the University of Arizona and Knowledge Computing Corporation for their support.

\section{References}

1. R. Adderley and P.B. Musgrove (2001). Data Mining Case Study: Modeling the Behavior of Offenders Who Commit Serious Sexual Assaults. In Proceedings of the Seventh ACM SIGKDD International Conference on Knowledge Discovery and Data Mining, San Francisco, California, August 26-29, 2001.

2. R. Armstrong, D. Freitag, T. Joachims and T. Mitchell (1995). WebWatcher: A Learning Apprentice for the World Wide Web. In Proceedings of the AAAI Spring Symposium on Information Gathering from Heterogeneous, Distributed Environments, Mar 1995.

3. M. Balabanovic and Y. Shoham (1997). Fab: Content-Based, Collaborative Recommendation. Communications of the ACM, 97(3), 66-72.

4. M. Brown (1998). Future Alert Contact Network: Reducing Crime Via Early Notification. http://pti.nw.dc.us/solutions/solutions98/public_safety/charlotte.html.

5. M. Chau, H. Atabakhsh, D. Zeng, and H. Chen (2001). Building an Infrastructure for Law Enforcement Information Sharing and Collaboration: Design Issues and Challenges. In Proceedings of the National Conference for Digital Government Research, Los Angeles, California, May 21-23, 2001.

6. M. Chau, D. Zeng, H. Chen, M. Huang, and D. Hendriawan (2003). Design and Evaluation of a Multi-agent Collaborative Web Mining System. Decision Support Systems, Special Issue on Web Retrieval and Mining, 35(1), 167-183.

7. H. Chen, J. Schroeder, R.V. Hauck, L. Ridgeway, H. Atabakhsh, H. Gupta, C. Boarman, K. Rasmussen, A. W. Clements (2003). COPLINK Connect: Information and Knowledge Management for Law Enforcement. Decision Support Systems, 34(3), 271-285.

8. J.P. Chin, V.A. Diehl, and K.L. Norman (1988). Development of an Instrument Measuring User Satisfaction of the Human-Computer Interface. In Proceedings of the ACM CHI '88, Washington, DC, pp. 213-218.

9. A. Dix, J. Finlay, G. Abowd, and R. Beale (1998). Human Computer Interaction. Englewood Cliffs, NJ: Prentice Hall.

10. R. Dussault (2000). Maps and Management: Comstat Evolves. Government Technology Crime and the Tech Effect, April 2000. 
11. M. Ginsburg (1998). Annotate! A Tool for Collaborative Information Retrieval. In Proceedings of the 7th IEEE International Workshop on Enabling Technologies: Infrastructure for Collaborative Enterprises (WET ICE'98), IEEE CS, Los Alamitos, California, 1998, 75-80.

12. D. Goldberg, D. Nichols, B. Oki and D. Terry (1992). Using Collaborative Filtering to Weave an Information Tapestry. Communications of the ACM, 35(12), 61-69.

13. M. Hansen (2002). Knowledge Networks: Explaining Effective Knowledge Sharing in Multiunit Companies. Organization Science, 13(3), 232-248.

14. R.V. Hauck and H. Chen (1999). COPLINK: A Case of Intelligent Analysis and Knowledge Management. In Proceedings of the 20th Annual International Conference on Information Systems, Charlotte, December 13-15, 1999.

15. M. J. Hoogeveen and K. van der Meer (1994). Integration of Information Retrieval and Database Management in Support of Multimedia Police Work. Journal of Information Science, 20(2), 79-87.

16. P.B. Kantor, E. Boros, B. Melamed, V. Meñkov, B. Shapira, and D.J. Neu (2000). Capturing Human Intelligence in the Net. Communications of the ACM, 43(8), 112-115.

17. J.A. Konstan, B. Miller, D. Maltz, J. Herlocker, L. Gordon and J. Riedl (1997). GroupLens: Applying Collaborative Filtering to Usenet News. Communications of the ACM, 40(3), 77-87.

18. B. Miller (1996). Searchable Databases Help Missouri Solve Crime. Government Technology, 9(8), 18-19.

19. D.E. O'Leary (1998). Knowledge Management Systems: Converting and Connecting. IEEE Intelligent Systems, 13(2), 30-33.

20. W. Orlikowski (1992). Learning from Notes: Organizational Issues in Groupware Implementation. In Proceedings of the ACM Conference on Computer Supported Cooperative Work (CSCW'92), 1992, 362-369.

21. N. Romano, D. Roussinov, J. F. Nunamaker, and H. Chen (1999). Collaborative Information Retrieval Environment: Integration of Information Retrieval with Group Support Systems. In Proceedings of the 32nd Hawaii International Conference on System Sciences (HICSS-32), 1999.

22. U. Shardanand and P. Maes (1995). Social Information Filtering: Algorithms for Automating "Word of Mouth." In Proceedings of the ACM Conference on Human Factors and Computing Systems, Denver, Colorado, May 1995.

23. B. Starr, M. Ackerman, and M. Pazzani (1996). Do-I-Care: A Collaborative Web Agent. In Proceedings of the ACM Conference on Human Factors in Computing Systems (CHI'96), 273-274.

24. J. Yen, A. Chung, H. Ho, B. Tam, R. Lau, M. Chau, K. Hwang (1999). Collaborative and Scalable Financial Analysis with Multi-agent Technology. In Proceedings of the $32^{\text {nd }}$ Hawaii International Conference on System Sciences, Maui, Hawaii, January 5-8, 1999.

25. D. Zeng, M. Dror, and H. Chen (2002). Efficient Scheduling of Periodic Information Monitoring Requests. Submitted to INFORMS Journal on Computing. 